\title{
The Effect of Comorbidity on the Prognosis of Acute Lung Injury and Acute Respiratory Distress Syndrome
}

\author{
Katsutoshi Ando ${ }^{1}$, Tokuhide Doi ${ }^{2}$, Sandra Y. Moody ${ }^{3}$, Yoshihiro Ohkuni ${ }^{1}$, \\ Sachiko Sato ${ }^{4}$ and Norihiro Kaneko ${ }^{1}$
}

\begin{abstract}
Objective We conducted a retrospective study assessing the relationship between comorbidity, using the Charlson Comorbidity Index (CCI), and the prognoses of acute lung injury (ALI) and acute respiratory distress syndrome (ARDS) patients.

Methods We analyzed the data of 47 patients with ALI and ARDS who were admitted to our center between April 2004 and July 2009. The patients were classified into 2 groups (survival and non-survival) 3 months after diagnosis, and demographic and clinical characteristics were analyzed. We also evaluated the ROC curve and Akaike's information criterion (AIC) to determine the most appropriate cut-off level for the $\mathrm{CCI}$ at 3 months survival. The survival rate was estimated based on the AIC results.

Results The mean age was 71.0 years; 25 (53\%) of the patients died within 3 months of the diagnosis. Although age, etiology of ALI and ARDS, and APACHE II score did not differ between the two groups, smoking history, CCI, SOFA score, and steroid use were higher in the non-survival group than in the survival group. Age was not significantly correlated with CCI; however, CCI had weak, but statistically significant correlations with the APACHE II and SOFA scores ( $r=0.387, p<0.01$ and $r=0.288, p<0.05$, respectively). AIC analysis revealed that a score of 4 on the CCI was the most appropriate cut off level for 3 months survival. The 3-month survival rate was lower in patients with a $\mathrm{CCI} \geq 4$ than in those with a $\mathrm{CCI}<4$ (9.5\% vs. $55.5 \%$, $\mathrm{p}<0.05)$.

Discussion This study showed that the prognosis of ALI and ARDS was affected more by comorbidity than by age, and that the CCI was useful for assessing patient comorbidities in ALI and ARDS. We have to consider that patients with a CCI score of 4 or more are at risk of developing multi-organ failure and have a poor prognosis.
\end{abstract}

Key words: acute lung injury, acute respiratory distress syndrome, charlson comorbidity index, comorbidity

(Intern Med 51: 1835-1840, 2012)

(DOI: 10.2169/internalmedicine.51.6434)

\section{Introduction}

Acute lung injury (ALI) and acute respiratory distress syndrome (ARDS), initially described by Ashbaugh et al., are rapidly progressive pulmonary syndromes that lead to respiratory failure, and are major contributors to mortality and morbidity in intensive care units (1). Recently, the prognosis of ALI and ARDS have improved due to the increasing use of ventilation with lower tidal volumes (TV) and high positive end-expiratory pressure (PEEP), the innovation of ventilation modes such as high-frequency ventilation or airway pressure release ventilation (APRV), and the widespread use of nutrition management including enteral feeding (2-6). Despite these advances, the prognosis remains poor and mortality rates high, approximately $20-50 \%(2,3)$. Moreover, we are facing an aging society in which the average life span is over 80 years. Because it has been reported that age and comorbidity are associated with a poor prognosis of ALI and ARDS $(7,8)$, it is predicted that the preva-

${ }^{1}$ Department of Pulmonology, Kameda Medical Center, Japan, ${ }^{2}$ Fukuoka Clinic, Japan, ${ }^{3}$ Department of Medicine, Division of Geriatrics, University of California, USA and ${ }^{4}$ Department of Anesthesiology, Kameda Medical Center, Japan

Received for publication August 24, 2011; Accepted for publication April 2, 2012

Correspondence to Dr. Katsutoshi Ando, ankatu1019@yahoo.co.jp 
Table 1. Charlson Comorbidity Index

\begin{tabular}{|c|c|}
\hline Points & Comorbidities \\
\hline 1 & $\begin{array}{l}\text { (1) Myocardial infarction } \\
\text { (2) Congestive heart failure } \\
\text { (3) Peripheral vascular Disease } \\
\text { (4) Dementia } \\
\text { (5) Chronic pulmonary disease } \\
\text { (6) Ulcer disease } \\
\text { (7) Mild liver disease } \\
\text { (8) Diabetes (without complications) } \\
\text { (9) Cerebrovascular disease }\end{array}$ \\
\hline 2 & $\begin{array}{l}\text { (1) Diabetes with end-organ damage } \\
\text { (2) Hemiplegia } \\
\text { (3) Moderate or severe renal disease } \\
\text { (4) Second solid tumor (nonmetastatic) } \\
\text { (5) Leukemia } \\
\text { (6) Lymphoma, multiple myeloma }\end{array}$ \\
\hline 3 & Moderate or severe liver disease \\
\hline 6 & $\begin{array}{l}\text { (1) Second metastatic solid tumor } \\
\text { (2) Acquired immunodeficiency syndrome }\end{array}$ \\
\hline
\end{tabular}

lence of ALI and ARDS among elderly patients with comorbidities will increase concomitantly with mortality.

The Charlson Comorbidity Index (CCI) is one of many recently published scales that measure comorbidities (9). This index involves giving weighted scores to 18 conditions. The sum of the scores is then scaled to establish the CCI staging system (Table 1). This index has been validated in, and used to predict the prognosis of pulmonary diseases such as lung cancer (10), interstitial pneumonia (11), and lung abscess (12). To our knowledge, however, a relationship between comorbidity and the prognosis of ALI and ARDS has never been reported. Thus, we conducted a retrospective analysis using CCI to assess the relationship between comorbidity and the prognosis of ALI and ARDS.

\section{Materials and Methods}

This retrospective study was approved by the ethics committees of our institution. We screened all mechanically ventilated patients with a diagnosis of ALI or ARDS, who were admitted to the medical intensive care unit at Kameda Medical Center between April 2004 and July 2009. Data were drawn from the electronic health records. The diagnosis of ALI and ARDS was validated upon review of relevant clinical data and chest radiographs of each patient by one critical care and one respiratory physician. The diagnoses of ALI and ARDS were based on the following criteria: acute onset; arterial hypoxemia with a $\mathrm{PaO}_{2} / \mathrm{FiO}_{2}$ ratio lower than 300 $\left(200<\mathrm{PaO}_{2} / \mathrm{FiO}_{2}<300\right.$, or $\mathrm{PaO}_{2} / \mathrm{FiO}_{2}<200$ was defined as ALI or ARDS, respectively, regardless of PEEP level); bilateral infiltrates seen on chest radiograph; and pulmonary artery occlusive pressure lower than $18 \mathrm{mmHg}$ or lack of clinical evidence of elevated left atrial pressure (13). We excluded patients for whom a diagnosis of heart failure could not be confirmed. Forty-seven patients with ALI and ARDS were included in the study and stratified by survival and nonsurvival status 3 months after diagnosis. Patient characteristics, comorbid conditions, ventilation setting and treatment were included in the analysis. To assess their comorbidities, we estimated the CCI stage at the time the patient met the diagnostic criteria of ALI and ARDS.

We used $\chi^{2}$ test for categorical data and Student's $t$ test for means and standard deviations to analyze the results with SPSS Statistics Version 19. Correlations of the disease severity (APACHE II and SOFA score), age and CCI were evaluated using Spearman's rank correlation analysis. Survival rate was estimated using the Kaplan-Meier method. For all statistical analyses, $\mathrm{p}$ value less than 0.05 was considered significant. Data were expressed as percentages and mean \pm standard deviation. To determine the most appropriate cut-off level of CCI for 3 months survival, we used the receiver operating characteristic (ROC) curve and Akaike's information criterion (AIC), which is an indicator for evaluating the goodness of fit of a statistical model $(14,15)$. A smaller AIC value indicated a more reliable model for predicting the outcome.

\section{Results}

\section{Patients' characteristics}

The median follow-up for subjects was 198.2 days (6.6 months). Mean age was 71.0 \pm 12.4 years. The most common etiologies of ALI and ARDS were pneumonia (31.9\%), sepsis $(48.9 \%)$, and unknown $(10.6 \%)$. The other less common causes were pneumothorax, pancreatitis, cholecystitis, and cerebrovascular disease $(2.1 \%$, respectively). Among the patients, 42 cases $(89.4 \%)$ also had comorbidities with a mean CCI of $2.49 \pm 1.54$. Twenty five out of $47(53.2 \%)$ patients did not survive three months after the diagnosis.

Age was not significantly correlated with CCI $(r=-0.051$, $\mathrm{p}=0.733)$, APACHE II $(\mathrm{r}=-0.013, \mathrm{p}=0.93)$ and SOFA score $(\mathrm{r}=-0.016, \mathrm{p}=0.44)$. However, CCI was statistically significantly correlated with APACHE II and SOFA score ( $\mathrm{r}=$ $0.387, p<0.01$ and $r=0.288, p<0.05$, respectively). Fig. 1 reveals the distribution of APACHE II and SOFA score accordingly to the CCI. These scores have a consistently positive correlation with CCI.

\section{Comparison between survival and non-survival cases}

The patients' characteristics stratified by survival and nonsurvival status are shown in Table 2. Although age, etiology of ARDS and ALI, and APACHE II scores did not differ between the two groups, smoking history (pack-year 56.3 vs. $12.3, \mathrm{p}<0.05)$, CCI (3.08 vs. $1.82, \mathrm{p}<0.01)$, SOFA score (7.44 vs. $5.23, \mathrm{p}<0.01)$, and steroid use $(24.0$ vs. $9.0 \%, \mathrm{p}<$ 0.01 ) were higher in the non-survival group than in the survival group. The comparison of the comorbidities is shown in Table 3; there was no significant difference between the groups.

\section{Overall survival and $\mathrm{CCl}$ staging}

Three-month survival was significantly lower in patients 
A

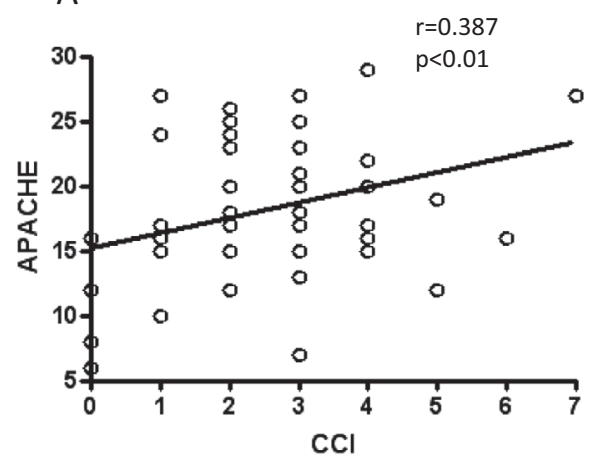

B

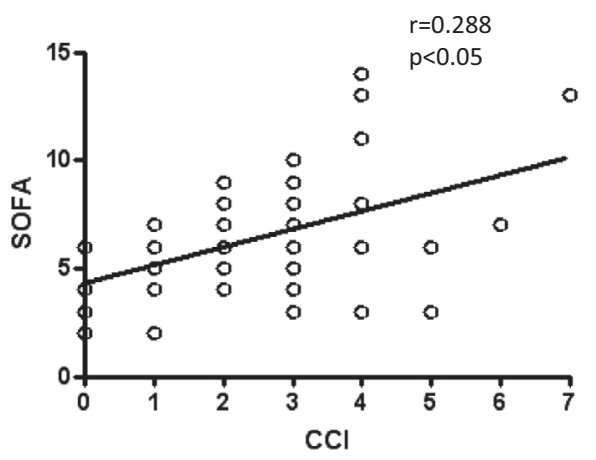

Figure 1. Correlation of disease severity with Charlson Comorbidity Index (CCI). APACHE II denotes acute physiology and chronic health evaluation, and SOFA sequential organ failure assessment. CCI was statistically significantly correlated with APACHE II and SOFA score (r=0.387, $\mathrm{p}<0.01$ and $\mathrm{r}=0.288, \mathrm{p}<0.05$, respectively).

Table 2. Patient Characteristics according to Survival and Nonsurvival Status

\begin{tabular}{|c|c|c|c|}
\hline Characteristics & $\begin{array}{c}\text { Survival } \\
(n=22)\end{array}$ & $\begin{array}{c}\text { Non-survival } \\
(n=25)\end{array}$ & p value \\
\hline \multicolumn{4}{|l|}{ Demographics } \\
\hline Age - yr & $68.8 \pm 14.8$ & $73.5 \pm 9.5$ & $\mathrm{p}=0.25$ \\
\hline \multicolumn{4}{|l|}{ Sex - no. $(\%)$} \\
\hline Men & $14(63.6)$ & $16(64.0)$ & $\mathrm{p}=1.0$ \\
\hline Women & $8(36.4)$ & $9(36.0)$ & \\
\hline Pack-year & $12.3 \pm 19.8$ & $56.3 \pm 52.5$ & $\mathrm{p}<0.05$ \\
\hline \multicolumn{4}{|l|}{ Clinical Measures $^{\dagger}$} \\
\hline APACHE II score & $17.0 \pm 6.4$ & $19.2 \pm 4.9$ & $\mathrm{p}=0.18$ \\
\hline SOFA score & $5.2 \pm 1.5$ & $7.4 \pm 2.5$ & $\mathrm{p}<0.01$ \\
\hline $\begin{array}{l}\text { Comorbid Conditions - } \\
\text { no. }(\%)\end{array}$ & $18(81.8)$ & $24(96.0)$ & $\mathrm{p}=0.17$ \\
\hline $\mathrm{CCI}$ & $1.82 \pm 1.15$ & $3.08 \pm 1.60$ & $\mathrm{p}<0.05$ \\
\hline $\mathrm{PaO}_{2} / \mathrm{FiO}_{2}$ ratio & $142.1 \pm 65.4$ & $113.3 \pm 38.6$ & $\mathrm{p}=0.07$ \\
\hline \multicolumn{4}{|l|}{ Incipient diseases ${ }^{\ddagger}-$ no. $(\%)$} \\
\hline Sepsis & $7(31.8)$ & $8(32.0)$ & $\mathrm{p}=1.0$ \\
\hline Pneumonia & $11(50.0)$ & $12(48.0)$ & $\mathrm{p}=1.0$ \\
\hline Pneumothorax & 0 & $1(4.0)$ & $\mathrm{p}=1.0$ \\
\hline Pancreatitis & $1(4.5)$ & 0 & $\mathrm{p}=0.47$ \\
\hline Cholecystitis & $1(4.5)$ & 0 & $\mathrm{p}=0.47$ \\
\hline Cerebral bleeding & $1(4.5)$ & 0 & $\mathrm{p}=0.47$ \\
\hline Unknown & $1(4.5)$ & $4(16.0)$ & $\mathrm{p}=0.35$ \\
\hline \multicolumn{4}{|l|}{ Ventilator setting $^{\dagger \dagger}$} \\
\hline Mean PEEP & $10.61 \pm 4.19$ & $10.26 \pm 5.46$ & $\mathrm{p}=0.83$ \\
\hline Mean Plateau P & $25.35 \pm 6.13$ & $21.67 \pm 6.88$ & $\mathrm{p}=0.10$ \\
\hline \multicolumn{4}{|l|}{ Ventilator mode $^{\dagger f}$-no. (\%) } \\
\hline Assist control & $14(63.6)$ & $16(64.0)$ & $\mathrm{p}=1.0$ \\
\hline APRV & 0 & $1(4.0)$ & $\mathrm{p}=1.0$ \\
\hline SIMV & $2(9.0)$ & $4(16.0)$ & $\mathrm{p}=0.67$ \\
\hline CPAP & $6(27.3)$ & $4(16.0)$ & $\mathrm{p}=0.47$ \\
\hline \multicolumn{4}{|l|}{ Treatment - no. $(\%)$} \\
\hline Antibiotics & $22(100)$ & $24(96.0)$ & $\mathrm{p}=0.90$ \\
\hline Steroids & $2(9.0)$ & $11(44.0)$ & $\mathrm{p}<0.01$ \\
\hline MP pulse & $2(9.0)$ & $6(24.0)$ & $\mathrm{p}=0.17$ \\
\hline Sivelestat & $1(4.5)$ & $1(4.0)$ & $\mathrm{p}=0.93$ \\
\hline
\end{tabular}

Plus-minus values are means $\pm \mathrm{SD}$.

${ }^{\dagger}$ Abbreviations used are: APACHE, acute physiology and chronic health evaluation; SOFA, sequential organ failure assessment; CCI, Charlson comorbidity index; $\mathrm{PaO}_{2} / \mathrm{FiO}_{2}$, partial pressure of oxygen/fractional inspired oxygen (normal range of $\mathrm{PaO}_{2} / \mathrm{FiO}_{2}$ ratio is $300-500 \mathrm{mmHg}$ ); PEEP, positive end-expiratory pressure; Plateau P, plateau pressure; APRV, Airway pressure release ventilation; SIMV, synchronized intermittent mandatory ventilation; CPAP, continuous positive airway pressure.

Diseases that led to the development of ALI and ARDS.

The values indicated are at the time of diagnosis of ALI and ARDS.

${ }^{\top}$ MP pulse: methylprednisolone (MP) pulse therapy which is treated by 3 days of MP $1,000 \mathrm{mg} /$ day. 
Table 3. Comorbid Conditions according to Survival and Non-survival Status

\begin{tabular}{lrrc}
\hline Characteristic & $\begin{array}{c}\text { Survival } \\
(\mathbf{n = 2 2})\end{array}$ & $\begin{array}{c}\text { Non-survival } \\
(\mathbf{n}=\mathbf{2 5})\end{array}$ & p value \\
\hline Diabetes mellitus & $3(14)$ & $6(24)$ & 0.47 \\
$\quad$ (with complication) & $0(0)$ & $1(4)$ & 0.90 \\
Cardiovascular disease & $3(14)$ & $9(36)$ & 0.10 \\
Peripheral vascular disease & $1(5)$ & $2(8)$ & 1.00 \\
Chronic pulmonary disease & $5(23)$ & $7(28)$ & 0.75 \\
Chronic kidney disease & $3(14)$ & $6(24)$ & 0.47 \\
Cerebrovascular disease & $3(14)$ & $1(4)$ & 0.33 \\
Gastrointestinal disease & $0(0)$ & $4(16)$ & 0.11 \\
Liver disease & $2(10)$ & $3(12)$ & 1.00 \\
Solid tumor & $5(23)$ & $8(32)$ & 0.53 \\
Hematologic disease & $1(5)$ & $4(16)$ & 0.35 \\
Collagen disease & $5(23)$ & $4(16)$ & 0.19 \\
\hline
\end{tabular}

with a CCI over 3.5 than in patients with lower scores (Fig. 2A), with an area under the ROC curve of $0.725 \pm$ 0.073 (95\% CI, 0.582-0.869). Moreover, to determine the most appropriate cut-off level of the CCI in detail, we calculated the AIC value, with a smaller AIC value indicating a more reliable prediction. As a result, the smallest AIC value of CCI was 4 (AIC=-5.85) (Fig. 2B). Based on the AIC statistics, the patients were divided into $\mathrm{CCI} \geq 4$ and $\mathrm{CCI}<4$. As a result, the 3-month survival rate was lower in patients with $\mathrm{CCI} \geq 4$ compared with those who had a $\mathrm{CCI}<4$ (9.5 vs. $55.5 \%$, p $<0.05$ ) (Fig. 2C).

\section{Discussion}

This retrospective analysis of 47 patients with ALI and ARDS showed that the CCI and SOFA score were higher in patients in the non-survival group compared to those in the survival group, even though age was not statistically different. The SOFA score is a scoring system that measures dysfunction across six organ systems -respiratory, cardiovascular, hepatic, coagulation, renal, and neurological- and can be used to assess the patient's status daily, assigning a score from 0 to 24 points. Sequential assessment of organ dysfunction during the first few days of ICU admission is a good indicator of prognosis (16). In this analysis, we additionally found that not only the SOFA but also APACHE II scores, a severity-of-disease classification system in which higher scores correspond to more severe disease and a higher risk of death (17), had significant positive correlations with CCI, while age had no correlation with these scores. These data indicate that patients with a high CCI are more likely to develop multi-organ failure and to be at risk for increased mortality. Recently, many countries face an aging society with a commensurate increase in elderly patients that have multiple comorbidities. In Japan, the average life expectancy has increased to over 80 years, which means the number of elderly patients with comorbidities will also increase. Davidson et al. reported that age and comorbidity are associated with survival in ARDS (8). The present results suggested, however, that the prognosis was influenced more by comorbidities than age. Accordingly, we suggest not only assessing age but also comorbidities using a concrete index.

In ALI and ARDS, a relationship between prognosis and comorbidity, using a concrete index to measure comorbidities has never been reported. Therefore, in patients with multiple comorbidities, it is difficult to determine whether comorbidities or the severity of ALI or ARDS influence prognosis the most. Given that, patients with multiple comorbidities are likely to increase, the assessment and validation of comorbidities in the setting of ALI and ARDS should be considered in clinical studies or trials. Recently, the CCI has been identified as the scale of choice for assessing comorbidities and prognostic factors of lung cancer, interstitial pneumonia, and lung abscesses (10-12). The present data revealed that the CCI had significant positive correlations with APACHE II and SOFA scores, and AIC analysis revealed that patients with a CCI greater than 4 tend to have multiple organ failure and a more severe case of ALI and ARDS. Our results indicate that CCI was useful for assessing comorbidities in patients with ALI and ARDS, and that it should be considered among the comorbidity scales included in clinical trials of ALI and ARDS.

Some clinical trials have reported the use of drugs such as glucocorticoid, protease inhibitors (sivelestat), and anticoagulant therapy in the treatment of ALI and ARDS (18-23). A few randomized controlled trials reported that methylprednisolone is associated with a reduction in the duration of mechanical ventilation, shock, and ICU length of stay $(18,19)$. Meduri et al. reported that the downregulation of systemic inflammation by methylprednisolone is associated with significant improvement in pulmonary and extrapulmonary organ dysfunction (18). In contrast to these results, however, it has been reported that methylprednisolone is associated with increased 60-day and 180-day mortality among patients enrolled at least 14 days after the onset of illness (19). Consequently, recent clinical practice guidelines for ALI and ARDS stated that although there was a possibility of reduced mortality and increased ventilatorfree days with steroids, the mortality benefits had never been established (24). On the other hand, the present data revealed that steroid use was higher in patients who did not survive compared to those who did, even though treatment with drugs such as sivelestat or antibiotics were not different. Due to the retrospective nature of the current study, it is unclear whether steroid use or the use of many additional drugs in patients who died was associated with increased mortality. Accordingly, we believe that our data did not suggest that steroid use is associated with increased mortality, but this needs to be confirmed by an adequately powered randomized clinical trial.

The present study has two major limitations. First, our study was a retrospective analysis and the number of patients included was relatively small. Second, the inciting diseases that our patients had may represent only a small sample of the various etiologies of ALI and ARDS. Since we only included patients that were admitted to the medical ICU, patients with trauma were not included. However, our 
A

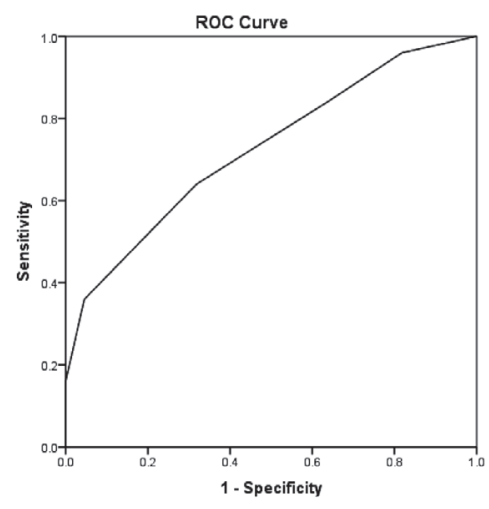

B
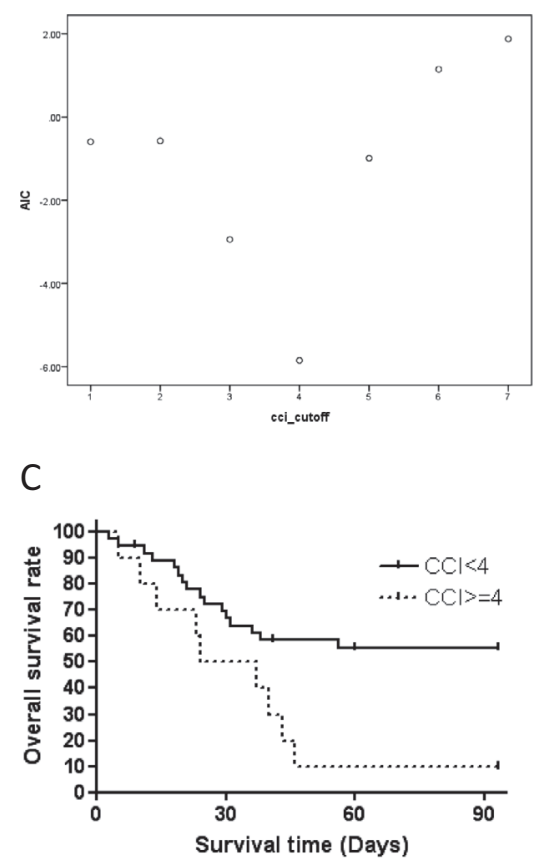

Figure 2. The relationship between Charlson Comorbidity Index (CCI) and 3-month survival. The area under the ROC curve $(0.725 \pm 0.073(95 \%$ CI, 0.582-0.869)) based on the patients' CCI (Panel A). The CCI of 4 was determined by AIC analysis (AIC = -5.85; also see Methods) to identify the most appropriate cut-off level for the discrimination between non-survival and survival cases (Panel B). Panel $\mathrm{C}$ showed the Kaplan-Meier estimate of survival in patients with a $\mathrm{CCI} \geq 4$ or $\mathrm{CCI}<4$. The 3-month survival rate was lower in patients with a $\mathrm{CCI} \geq 4$ than in those with a $\mathrm{CCI}<4$ (9.5\% vs. 55.5 $\%, \mathbf{p}<0.05)$.

results showed that having multiple comorbidities influenced the prognosis of ALI and ARDS. Therefore, in this context, we should re-examine our results prospectively in a multicenter.

In conclusion, this study showed that the prognosis of ALI and ARDS was influenced more by comorbidity than by age. Consistent with our findings, patients with a CCI of greater than 4, and who have ALI or ARDS, are more likely to develop multi organ failure and have a poor prognosis.

The authors state that they have no Conflict of Interest (COI).

\section{References}

1. Ashbaugh DG, Bigelow DB, Petty TL, Levine BE. Acute respiratory distress in adults. Lancet 290: 319-323, 1967.

2. Brower RG, Lanken PN, Maclntyre N, et al. National Heart, Lung and Blood Institute ARDS Clinical Trials Network: Higher versus lower positive end expiratory pressures in patients with acute respiratory distress syndrome. N Engl J Med 22: 327-336, 2004.

3. The Acute Respiratory Distress Syndrome Network. Ventilation with lower tidal volumes as compared with traditional tidal volumes for acute lung injury and the acute respiratory distress syndrome. N Engl J Med 342: 1301-1308, 2000.

4. Burchardi $\mathrm{H}$. New strategies in mechanical ventilation for acute lung injury. Eur Respir J 9: 1063-1072, 1996.

5. Derdak S, Mehta S, Stewart TE, et al. Multicenter oscillatory ventilation for acute respiratory distress syndrome. Am J Respir Crit Care Med 166: 801-808, 2002.
6. Gadek JE, DeMichele SJ, Karlstad MD, et al. Effect of enteral feeding with eicosapentaenoic acid, gamma-linolenic acid, and antioxidants in patients with acute respiratory distress syndrome. Enteral Nutrition in ARDS study group. Crit Care Med 27: 14091420, 1999.

7. Ely EW, Wheeler AP, Thompson BT, Ancukiewicz M, Steinberg $\mathrm{KP}$, Bernard GR. Recovery rate and prognosis in older persons who develop acute lung injury and the acute respiratory distress syndrome. Ann Intern Med 136: 25-36, 2002.

8. Davidson TA, Rubenfeld GD, Caldwell ES, Hudson LD, Steinberg KP. The effect of acute respiratory distress syndrome on long-term survival. Am J Respir Crit Care Med 160: 1838-1842, 1999.

9. Charlson ME, Pompei P, Ales KL, MacKenzie CR. A new method of classifying prognostic comorbidity in longitudinal studies: development and validation. J Chronic Dis 40: 373-383, 1987.

10. Asmis TR, Ding K, Seymour L, et al. Age and comorbidity as independent prognostic factors in the treatment of non small-cell lung cancer: a review of National Cancer Institute of Canada Clinical Trials Group Trials. J Clin Oncol 26: 54-59, 2008.

11. Ando K, Ohkuni Y, Makino H, Kawamura Y, Motojima S, Kaneko $\mathrm{N}$. The relationship between the prognosis of interstitial pneumonia and its comorbidities. Nihon Kokyuki Gakkai Zasshi 49: 800809, 2011 (in Japanese, abstract in English).

12. Ando K, Ohkuni $Y$, Matsunuma R, et al. Prognostic lung abscess factors. Kansenshogaku Zasshi 84: 425-430, 2010 (in Japanese, abstract in English).

13. Akaike H. A new look at statistical model identification. IEEE Trans. Automat Contr 19: 716-723, 1974.

14. Wagenmakers EJ, Farrell S. AIC model selection using Akaike weights. Psychon Bull Rev 11: 192-196, 2004.

15. Hines RB, Chatla C, Bumpers HL, et al. Predictive capacity of three comorbidity indices in estimating mortality after surgery for 
colon cancer. J Clin Oncol 27: 4339-4345, 2009.

16. Vincent JL, Moreno R, Takala J, et al. The SOFA (Sepsis-related Organ Failure Assessment) score to describe organ dysfunction/ failure. On behalf of the working group on sepsis-related problems of the european society of intensive care medicine. Intensive Care Med 22: 707-710, 1996.

17. Knaus WA, Draper EA, Wagner DP, Zimmerman JE. APACHE II: a severity of disease classification system. Critical Care Medicine 13: 818-829, 1985.

18. Meduri GU, Golden E, Freire AX, et al. Methylprednisolone infusion in early severe ARDS: results of a randomized controlled trial. Chest 131: 954-963, 2007.

19. The National Heart, Lung, and Blood Institute Acute Respiratory Distress Syndrome (ARDS) Clinical Trials Network. Efficacy and safety of corticosteroids for persistent acute respiratory distress syndrome. N Engl J Med 354: 1671-1684, 2006.
20. Tang BM, Craig JC, Eslick GD, Seppelt I, McLean AS. Use of corticosteroids in acute lung injury and acute respiratory distress syndrome: a systematic review and meta-analysis. Crit Care Med 37: 1594-1603, 2009.

21. Peter JV, John P, Graham PL, Moran JL, George IA, Bersten A. Corticosteroids in the prevention and treatment of acute respiratory distress syndrome (ARDS) in adults: meta-analysis. BMJ 336: 1006-1009, 2008.

22. STRIVE Study Group. Neutrophil elastase inhibition in acute lung injury: results of the STRIVE study. Crit Care Med 32: 16951702, 2004.

23. Laterre PF, Wittebole $\mathrm{X}$, Dhainaut JF. Anticoagulant therapy in acute lung injury. Crit Care Med 31(4 Suppl): S329-S336, 2003.

24. The Japanese Respiratory Society. Clinical practice guideline for acute lung injury and acute respiratory distress syndrome. 2nd. Shujunsha, Tokyo, 2010: 71-88.

(C) 2012 The Japanese Society of Internal Medicine http://www.naika.or.jp/imindex.html 